OPEN ACCESS

Citation: Francesca Borruso (2020) Juri Meda, I «Monumenta Italiae Paedagogica" e la costruzione del canone pedagogico nazionale (1886-1956). Rivista di Storia dell'Educazione 7(2): 153-155. doi: 10.36253/rse-10097

Received: December 4, 2020

Accepted: December 4, 2020

Published: January 25, 2021

Copyright: (c) 2020 Francesca Borruso. This is an open access, peer-reviewed article published by Firenze University Press (http://www.fupress.com/rse) and distributed under the terms of the Creative Commons Attribution License, which permits unrestricted use, distribution, and reproduction in any medium, provided the original author and source are credited.

Data Availability Statement: All relevant data are within the paper and its Supporting Information files.

Competing Interests: The Author(s) declare(s) no conflict of interest.

Editor: Pietro Causarano, Università di Firenze.
Recensione

\section{Juri Meda, I «Monumenta Italiae Paedagogica» e la costruzione del canone pedagogico nazionale (1886-1956)}

\author{
Milano, Franco Angeli, 2019, pp. 294
}

Francesca Borruso

Università di Roma 3

E-mail: francesca.borruso@uniroma3.it

Il libro di Juri Meda, che ha ricevuto nel 2020 il premio CIRSE dedicato alle migliori opere di carattere storico-educativo, colma una significativa lacuna storiografica inserendosi all'interno di una tradizione di ricerca consolidata sul piano internazionale ma, al contempo, innovativa e coraggiosa per la complessità e ricchezza delle fonti archivistiche utilizzate e per la molteplicità dei piani interpretativi coinvolti.

Attraverso il reperimento e l'analisi di fonti edite ma soprattutto inedite provenienti da una dozzina di archivi italiani (riprodotte nel volume all'interno di un'ampia appendice documentaria), l'Autore è riuscito a ricostruire, in modo dettagliato e rigoroso, il ricco e variegato dibattito pedagogicoculturale che ha accompagnato un ambizioso progetto editoriale italiano mai realizzato, quello dei Monumenta Italiae Pedagogica. Un progetto mancato nella sua realizzazione ma attorno al quale si sono condensate 'partite culturali' emblematiche, rivelatrici sia delle lotte egemoniche fra i diversi orientamenti pedagogici, sia delle trasformazioni relative allo statuto epistemologico costitutivo che si attribuiva al sapere storico-pedagogico. Così, attraverso la ricostruzione storico-pedagogica-culturale di un fallimento editoriale significativo, Juri Meda ha delineato, con prospettive euristiche innovative, le idee e le progettualità di una classe intellettuale certamente eterogenea al suo interno - anche in ragione del vasto periodo storico che la caratterizza (18861956) -, così come le conseguenze profonde e concrete che sulla storia della pedagogia italiana hanno avuto quelle vicende. Idee e progetti che si intersecano, inevitabilmente, con i complessi processi storico-sociali e culturali connessi all'unificazione nazionale e alla costruzione di una identità culturale vissuta come discorso imprescindibile e fondante l'unità della nazione.

$\mathrm{Al}$ progetto si dedicarono, seppure con prospettive culturali e in momenti storici differenti (da quelle di ispirazione positivista, all'orientamento neo-idealista e al pensiero cattolico), soprattutto due grandi pedagogisti che rispondono ai nomi di Luigi Credaro e Giovanni Calò accomunati, nono- 
stante le diverse prospettive pedagogiche, da un medesimo obiettivo: giungere alla definizione di un "canone pedagogico nazionale", ossia all'individuazione di quell'insieme di opere educative alle quali attribuire un valore esemplare, fondative di una tradizione educativa tutta italiana e che proprio per questo, andavano valorizzate e diffuse come patrimonio identitario irrinunciabile. Un canone pedagogico, inoltre, che avrebbe potuto esplicare i suoi effetti anche in relazione alla formazione della classe docente, responsabile a sua volta della costruzione di una consapevole identità nazionale all'interno delle istituzioni educative.

Un progetto che, proprio per i suoi propositi identitari ed epistemologici del discorso storico-pedagogico, secondo Juri Meda, già dai primordi avrebbe mostrato il suo positivo influsso rivelandosi anticipatore della crisi epistemologica della storia della pedagogia «intesa esclusivamente come storia delle idee pedagogiche e delle teorie educative, che sarebbe stata rilanciata a inizio del '900 dal neo idealismo e dall'egemonia da essa esercitata sull'intera cultura italiana», alimentando questo processo di trasformazione, «ben oltre la sua soglia naturale di obsolescenza e quando ormai nella storiografia internazionale si era diffuso il modello annalistico francese, che l'avrebbe più tardi influenzato» ( p. 19).

È Luigi Credaro uno dei primi a proporre, cercando di abbozzarne la struttura generale e definirne gli intenti fondamentali, il progetto italiano dei Monumenta, ispirandosi sì al modello tedesco dei Monumenta Germaniae Historica - imponente raccolta di fonti per la storia del medioevo tedesco ed europeo - ma con l'idea innovativa di valorizzare non solo la letteratura pedagogica italiana, per il filosofo non poi così meritevole e vasta da essere oggetto di inventario speciale, ma soprattutto «i tesori didattici, che stanno nascosti negli archivi e nelle biblioteche del nostro paese» (p. 52). Nell'appello che Credaro rivolge alla comunità scientifica italiana affinché promuova la costituzione nel nostro paese di una società simile a quella tedesca, sono «gli eruditi, i ricercatori di documenti, gli studiosi abitatori delle biblioteche e degli archivi» che «dovrebbero segnalare al pubblico quelle carte che interessano il passato dell'educazione del nostro paese» (p. 53). Quindi, più che una collezione di trattati educativi e opere pedagogiche, l'idea era quella di una raccolta sistematica di documenti relativi alla concretezza delle prassi educative della scuola e dell'educazione (storia delle istituzioni scolastiche, della legislazione educativa, dei metodi didattici applicati alle scuole, così come degli abbecedari e dei libri adottati), a partire soprattutto dalla modernità. Un'idea che si collocava perfettamente nel quadro del neo-herbartismo di inizio secolo, di cui Credaro era un illustre esponente, orien- tata a fondare una storia dell'educazione e della scuola non più ancillare alla storia filosofica bensì autonoma, $\mathrm{e}$ antitetica all'ascendente neoidealismo gentiliano il quale invece si opponeva all'autonomia epistemologica della pedagogia come scienza autonoma dalla filosofia e, di conseguenza, a qualunque ibridazione epistemologica con altri saperi. In questa ottica di contrasto alla deriva storicistica della storia della pedagogia che il neoidealismo combatte, può essere interpretata la creazione della collana «Pedagogisti ed educatori antichi e moderni», presso la casa editrice Sandron, diretta da Giuseppe Lombardo Radice dal 1910 al 1939, nella quale vengono pubblicate, con un apparato critico, le opere dei maggiori pedagogisti ed educatori antichi e moderni, incluse le opere «di insigni autori, comunemente non classificati fra i pedagogisti, ma che han lasciato tracce profonde nel campo educativo: poeti, storici, filosofi, agitatori» (p. 83). Una collana editoriale che secondo Juri Meda avrebbe potuto (o voluto?) rappresentare un'alternativa al progetto dei Monumenta - visto che nelle intenzioni programmatiche si vogliono ricostruire le grandi traiettorie del pensiero pedagogico secondo l'insegnamento neoidealistico - ma che sin dai suoi esordi assegna più visibilità alla cultura pedagogica internazionale più che a quella nazionale, al punto che nel primo anno di vita solo 8 volumi su 52 sono dedicati ad autori italiani.

Nella lotta per l'egemonia culturale fra i diversi orientamenti pedagogici, che Juri Meda ha saputo acutamente ricostruire, si colloca anche la figura di Giovanni Calò, teorico dello 'spiritualismo realistico' (alternativo all'herbartismo credariano), collaboratore della «Rivista Pedagogica» e impegnato con il suo maestro Francesco De Sarlo in uno scontro con il neoidealismo, il quale nel 1933 recupera il progetto dei Monumenta e lo propone alla Reale Accademia dei Lincei, probabilmente di concerto con Credaro, come sembra testimoniare la corrispondenza intercorsa tra Calò e Credaro, iniziata nel 1909 e conclusasi soltanto con la morte di Credaro nel 1939, già messa in luce da Giorgio Chiosso nel 1984. Nonostante gli sforzi di Calò, capace di muoversi con grande capacità diplomatica in un panorama politico-culturale complesso e non certo 'amico' - come riesce a documentare Meda ricostruendo i finanziamenti che Calò riesce a procurarsi per il Museo Didattico Nazionale di Firenze (1925-1929) - la prima richiesta di finanziamento all'impresa dei Monumenta venne rigettata. Instancabile, Calò la propose nuovamente all'Accademia dei Lincei nel 1940, con un piano di pubblicazione dettagliato che prevedeva una I serie centrata sugli Scrittori e una II serie centrata sulle Fonti documentarie, nonostante il contesto politico e culturale fosse ancora più nebuloso di prima, a causa sia del consolidamento 
dell'egemonia idealistica sia dell'intolleranza del regime verso quegli intellettuali, come Calò, che non mostravano esplicita adesione al regime. Dal discorso di Calò di presentazione del progetto dei Monumenta emerge l'idea di una storia della pedagogia: «che non poteva limitarsi a una storia della scienza dell'educazione, dei sistemi pedagogici, delle teorie, della vita dei singoli filosofi e pedagogisti, ma doveva estendere i propri indirizzi euristici, aprendosi $[\ldots]$ alla storia delle istituzioni educative e - sulla base dei primi segnali del rinnovamento storiografico che preludeva all'incessante ascesa della nouvelle histoire francese - alla storia della vita materiale della scuola» (p. 141).

Il progetto di Calò, monumentale poiché prevedeva di stampare oltre una sessantina di volumi, molti dei quali dedicati a figure o questioni storico-educative quasi inedite per quel tempo storico, nella individuazione del canone pedagogico restituiva centralità alle pedagogie umanistica, cristiana a partire dal medioevo, e risorgimentale. Assicurato dal Ministro dell'Educazione Nazionale Bottai un sostegno economico tramite la formula dell'acquisto copie dei volumi e nominata la Commissione dei lavori, la monumentale impresa venne ufficialmente avviata ma si interruppe forzatamente a causa dello scoppio della seconda guerra mondiale, per naufragare definitivamente nel 1956. Nonostante il loro fallimento, i Monumenta continuarono ad esercitare il proprio influsso sulla produzione storiografica successiva, come nel caso della raccolta de I classici della pedagogia italiana diretta da Ernesto Lama e Luigi Volpicelli realizzata tra il 1956 e il 1963. Un influsso che, secondo l'Autore può essere ritrovato anche in relazione alla svolta storiografica di matrice annalistica, che restituisce valore sia alla materialità educativa, sia alle fonti della 'vita privata' e a tutte quelle fonti che, 'loro malgrado' secondo la celebre definizione di Marc Bloch, possono concorrere a disvelare la complessità storico-sociale dei fenomeni. Una complessità che Meda ha contribuito a decifrare in questo suo ultimo lavoro, intrecciando sapientemente e con grande rigore epistemologico, storia delle idee pedagogiche con la storia sociale dei processi culturali. 